\title{
BMJ Open Protocol for a scoping review to identify and map the global health personnel considered skilled attendants at birth in low and middle-income countries between 2000 and 2015
}

\author{
Amy J Hobbs, ${ }^{1}$ Ann-Beth Moller, ${ }^{2}$ Liliana Carvajal-Aguirre, ${ }^{3}$ Agbessi Amouzou, ${ }^{1}$ \\ Doris Chou, ${ }^{2}$ Lale Say ${ }^{2}$
}

To cite: Hobbs AJ, Moller A-B, Carvajal-Aguirre L, et al. Protocol for a scoping review to identify and map the global health personnel considered skilled attendants at birth in low and middleincome countries between 2000 and 2015. BMJ Open 2017;7:e017229. doi:10.1136/ bmjopen-2017-017229

- Prepublication history and additional material are available. To view these files please visit the journal online (http://dx.doi org/10.1136/bmjopen-2017017229).

Received 8 April 2017

Revised 30 June 2017 Accepted 13 July 2017

Check for updates

${ }^{1}$ Department of International Health, The Institute for International Programs, Johns Hopkins Bloomberg School of Public Health, Baltimore, Maryland, USA

${ }^{2}$ Department of Reproductive Health and Research, World Health Organization, Geneva, Switzerland

${ }^{3}$ United Nations Children's Fund (UNICEF), New York City, New York, USA

Correspondence to

Dr Doris Chou; choud@who.int

\section{ABSTRACT}

Introduction Despite progress towards the Millennium Development Goals (MDG), maternal mortality remains high in countries where there are shortages of skilled personnel able to manage and provide quality care during pregnancy and childbirth. The 'percentage of births attended by skilled health personnel' (SAB, skilled attendants at birth) was a key indicator for tracking progress since the MDGs and is part of the Sustainable Development Goal agenda. However, due to contextual differences between and within countries on the definition of $S A B$, a lack of clarity exists around the training, competencies, and skills they are qualified to perform. In this paper, we outline a scoping review protocol that poses to identify and map the health personnel considered SAB in low and middle-income countries (LMIC).

Methods and analysis A search will be conducted for the years 2000-2015 in PubMed/MEDLINE, EMBASE, CINAHL Complete, Cochrane Database of Systematic Reviews, POPLINE and the WHO Global Health Library. A manual search of reference lists from identified studies or systematic reviews and a hand search of the literature from international partner organisations will be done. Original studies conducted in LMIC that assessed health personnel (paid or voluntary) providing interventions during the intrapartum period will be considered for inclusion.

Ethics and dissemination A scoping review is a secondary analysis of published literature and does not require ethics approval. This scoping review proposes to synthesise data on the training, competency and skills of identified $S A B$ and expands on other efforts to describe this global health workforce. The results will inform recommendations around improved coverage measurement and reporting of $S A B$ moving forward, allowing for more accurate, consistent and timely data able to guide decisions and action around planning and implementation of maternal and newborn health programme globally. Data will be disseminated through a peer-reviewed manuscript, conferences and to key stakeholders within international organisations.

\section{INTRODUCTION}

Despite progress towards Millennium Development Goal 4 and Goal 5 which aimed

\section{Strengths and limitations of this study}

- This scoping review proposes to identify and map the global health workforce considered skilled attendants at birth $(\mathrm{SAB})$ in low and middle-income countries. No other review has assessed $S A B$ on a global scale.

- The identified cadres considered SAB will be compared against internationally agreed-upon criteria as proposed in the 2004 WHO/ICM/FIGO (WHO/International Confederation of Midwives/ International Federation of Gynecology and Obstetrics) joint statement on the skilled attendant.

- The search strategy is broad and comprehensive, including 11 global electronic databases spanning both peer-reviewed and grey literature and does not have language restrictions.

Due to the broad nature of this review and inherent heterogeneity of data between and within countries, the final data extraction sheet and analysis plan are unable to be defined until after the review is completed.

to reduce maternal and child health and survival, ${ }^{1}$ mortality rates remain high in low and middle-income countries (LMIC) where there are critical shortages of health personnel who are able to adequately manage and provide quality care during pregnancy and childbirth. ${ }^{2-4}$ The Sustainable Development Goal (SDG) agenda highlights the importance of continued momentum towards improving maternal and newborn health by setting, under the SDG goal 3, targets for achieving a global maternal mortality ratio of less than 70 maternal deaths per 100000 live births, and aiming for all countries to reduce neonatal mortality to at least as low as 12 per 1000 live births by $2030 .^{56}$

A key progress indicator that is included in the SDG framework is the "percentage of 
births delivered by skilled attendant at birth' (SDG Indicator 3.1.2) ${ }^{67}$ It has also been identified as a core coverage indicator by other global monitoring frameworks, such as the Global Strategy for Women's, Adolescent's, and Children's Health ${ }^{8}$ Ending Preventable Maternal Mortality initiative $^{9}$ and Every Newborn Action Plan. ${ }^{10}$ According to the 2004 joint statement by the WHO, the International Confederation of Midwives (ICM) and the International Federation of Gynecology and Obstetrics (FIGO), a skilled attendant at birth $(\mathrm{SAB})$ is defined as: 'A midwife, doctor or nurse-who has been educated and trained to proficiency in the skills needed to manage normal (uncomplicated) pregnancies, childbirth and the immediate postnatal period, and in the identification, management and referral of complications in women and newborns. ${ }^{11}$

Despite guidance from the 2004 WHO/FIGO/ICM joint statement on the definition of a skilled attendant and its core functions, actual reporting at country level is challenged by lack of clear guidance on measurement standards, heterogeneity in the use of terminology and cadre functions. ${ }^{12}{ }^{13}$ Many countries have attempted to improve maternal health and survival through task shifting, by increasing the proportion of births attended by skilled health personnel through training lower level cadre, or creating new cadres able to provide pregnancy and childbirth care. ${ }^{14}$ However, the content and requirements of training programme may not be standardised, evaluated or publicly available. Even when there are practice standards and/or guidelines in place, many countries may lack the capacity and infrastructure needed to adhere to current recommendations for training and education. Thus, there may be confusion over what a SAB is, what they should be able to perform, how they should be trained and what systems should be in place in order to support them. ${ }^{15}$

The skill level and competencies of cadres may vary, and many cadres that are currently considered 'skilled' may not actually meet the internationally agreed-upon definition and criteria set in the $2004 \mathrm{WHO} /$ FIGO/ICM joint statement. ${ }^{12} 15$ The inclusion of additional country-level health personnel as skilled in global monitoring frameworks, without verification of training and capabilities, coupled with contextual differences between and within countries on the definition of what constitutes a SAB complicates the accuracy, comparability and consistency for continued measurement of SAB moving forward.

The aim of this scoping review is to identify and map the training, education, skill set and/or competency of the various cadres of health personnel that provided pregnancy and childbirth care in LMIC during 2000-2015. To our knowledge, no other review has been conducted on this topic previously in the published literature. This information will support the refinement of the definition of what constitutes a SAB in order to harmonise and improve the measurement around the global monitoring of SAB coverage and progress of the SDG targets set for $2030 .^{6}$

\section{METHODS AND ANALYSIS}

\section{Study design}

The study methods are based on frameworks developed for the design and conduct of systematic scoping reviews. ${ }^{16-19}$ Due to the broad and vast scope of our topic, a scoping review design was chosen in order to identify and map the existing literature. This protocol is conducted in accordance with the PRISMA-P (Preferred Reporting Items for Systematic Reviews and Meta-analysis Protocols) guideline ${ }^{20}$ as outlined in online supplementary additional file 1 . The final scoping review will follow the PRISMA reporting guidelines for systematic reviews. ${ }^{21}$

\section{Research questions}

The objectives of our study are to identify and map cadres considered SAB in relation to education, training, accreditation, certification, legislation, skills/competency; and/ or continuing education requirements in LMIC. The following protocol for a scoping review poses to address the following questions:

1. Who are the cadres of health personnel that are reported as 'skilled attendants at birth' as defined by the 2004 WHO/FIGO/ICM joint statement ${ }^{11}$ in LMIC?

2. How do these identified cadres differ between and within county in terms of the following?

a. curriculum, duration of training and/or education requirements obtained to be qualified;

b. regulation, accreditation and/or certification by a professional organisation;

c. skills and competency that each cadre is able to perform (ie, signal functions and/or other key interventions required for the management of childbirth);

d. legislation to perform these signal functions and/ or interventions;

e. location of work (urban/rural, hospital/ health centre/communitybased);

f. continuing education requirements (curriculum, duration, frequency).

\section{Inclusion criteria}

The aforementioned research questions will be assessed and studies will be selected specific to the following population, concept, study design and context criteria presented in table 1 .

All primary source study designs reporting on original human studies research will be included if it provides or compares the education and/or training received, accreditation or certification requirements, legislation, skills and/or competencies of the health personnel in order to be considered skilled attendants (paid or voluntary) who provide interventions related to the delivery of maternal and newborn health during pregnancy and childbirth (intrapartum). Secondary source data including systematic reviews and other study designs such as case reports, commentaries, editorials, letters or other opinion pieces will be excluded. Research articles reporting on health personnel providing pregnancy and childbirth care will 
Table 1 Inclusion criteria for identifying eligible studies

Inclusion criteria

\begin{tabular}{ll}
\hline Population & $\begin{array}{l}\text { Any health personnel (paid or voluntary) who provide health services within the provision of } \\
\text { maternal and newborn healthcare during pregnancy and childbirth }\end{array}$ \\
Concept & $\begin{array}{l}\text { Mapping of the health personnel according to education/training received; accreditation or } \\
\text { certification; legislation, skills/competency; and/or continuing education requirements }\end{array}$ \\
Study design & $\begin{array}{l}\text { Primary source research of any study design conducted on human subjects (observational studies } \\
\text { including prospective or retrospective cohort, case-control and case series; quasiexperimental, }\end{array}$ \\
experimental, and randomised controlled trials; and qualitative study designs)
\end{tabular}

be included regardless of if the cadres are formally regulated as SAB within a country and/or if they are legislated or not to perform key health interventions. The cadres included will be compared with the 2004 WHO/FIGO/ ICM joint statement in order to assess whether the cadre is considered skilled against the internationally agreedupon standard definition of a skilled attendant. ${ }^{11}$ Studies published inclusive of all languages, any study design and between the years 2000 and 2015 in LMIC countries will be considered eligible for inclusion.

\section{Exclusion criteria}

Articles will not be eligible for inclusion in the scoping review if:

1. There is no mention of the individual health personnel (cadre name) who are considered skilled attendants, providing maternal and newborn care during childbirth.

2. There are no details of at least one of the following key concepts:

a. education and/or training requirements that the cadre has received to be considered a skilled attendant;

b. if the cadre is formally accredited/certified within standard criteria set by the country;

c. legislation or regulatory requirements;

d. specific skills or key interventions/signal functions that the cadre is able to perform, regardless of whether they are legislated or authorised.

Personnel who provide supportive care during labour to the woman or act as a birth assistant, such as doulas, will not be considered skilled attendants for inclusion in this review.

Any study designs evaluating or comparing interventional training programmes will be excluded as the intent of our review is to map the training received by the cadre, not the content of new or existing training programmes.

A manual mapping of the cadres considered SAB within nationally representative household coverage surveys has been previously conducted by reviewing the Demographic Health Surveys (DHS) ${ }^{22}$ and Multiple Indicator Cluster Surveys (MICS) ${ }^{23}$ as part of a harmonised UNICEF and WHO joint database on $\mathrm{SAB}^{24}$ and has been conducted previously. ${ }^{25}$ Thus, studies that are a reanalysis of data from DHS or MICS will also be excluded.

\section{Search strategy}

The search strategy will be conducted for all relevant existing literature without language restrictions based on search terms relating to the research questions restricted to the years 2000-2015 using the following online bibliographic databases: PubMed/MEDLINE, EMBASE, CINAHL Complete, the Cochrane Database of Systematic Reviews, POPLINE and the WHO Global Health Library (GHL). The WHO GHL contains the following databases: African Index Medicus (AIM/AFRO); Latin American and Caribbean Health Sciences Information (LILACS/ AMRO/PAHO); Index Medicus for the Eastern Mediterranean Region (IMEMR/IMRO); the West Pacific Index Medicus (WPIM/WPRO); Index Medicus for the SouthEast Asian Region (IMSEAR/SEARO); and the WHO Library (WHOLIS).

A manual search of the reference lists of all identified studies or systematic reviews as well as a hand search of the literature from global initiatives for additional data, including UNFPA, WHO and UNICEF, will be done. In order to address which cadres are considered SAB worldwide and what skills they possess, how they are trained and how to best support these cadres, a search was conducted with specific terms and MeSH headings. An example of the full electronic search strategy to be conducted in PubMed/MEDLINE is outlined in Supplementary file 2 .

\section{Study selection}

Following the aforementioned comprehensive search strategy, article titles and abstracts will be screened and eligibility for inclusion assessed independently by three reviewers (AJH, ABM and LCA). Agreement regarding the identified abstracts will be quantified using the $\kappa$ statistic $^{26}$ and disagreement resolved by an additional reviewer $(\mathrm{DC})$.

Screened abstracts identified for inclusion will undergo an independent full-text review by three reviewers $(\mathrm{AJH}$, $\mathrm{ABM}$ and LCA). Identified non-English language full-text articles will be screened by native/advanced speakers to assess whether they meet our criteria for inclusion. Fulltext articles that have been excluded at the screening 
stage will have reasons for exclusion documented. The final chosen full-text studies will again be compared between the reviewers with disagreement being resolved by mutual consensus and with input from all coauthors. Those studies that do not meet the above criteria will be disqualified and removed from consideration. Authors from studies with unavailable or unclear data will be contacted to determine their eligibility for inclusion.

\section{Data extraction and analysis}

Data will be extracted from full-text journal articles, reports and other literature, which meet the inclusion criteria as outlined above. Data will be extracted using a Microsoft Excel database. Information retrieved will include study characteristics (country, region, years assessed, study objectives, methodology and study design). Details about the cadres identified include cadre name, if they are considered skilled (yes or no), education requirements (entry requirements, duration, content), continuing education (duration, content, frequency), ability to perform signal functions and/or other childbirth interventions (yes or no), number of normal or complicated deliveries performed over a defined time period, and if there are any regulatory bodies or legislation (yes or no) for the cadre named.

Data extracted regarding signal functions will include the seven basic and nine comprehensive services for emergency obstetric care as recommended by the WHO. ${ }^{27} 28$ The seven basic signal functions include: (1) administration of intravenous/intramuscular antibiotics; (2) administration of intravenous/intramuscular uterotonic drugs (ie, oxytocin); (3) administration of intravenous/intramuscular anticonvulsants; (4) manual removal of the placenta; (5) removal of retained products of conception; (6) performing assisted vaginal delivery; and (7) performing basic neonatal resuscitation. ${ }^{27} 28$ The two additional signal functions that comprise comprehensive services include: (8) performing caesarean section; and (9) performing blood transfusion. ${ }^{27} 28$

The data extraction form will contain additional fields to allow flexibility for the emergence of other themes and/ or categories, which will be discussed and developed via consensus from all coauthors. Resultantly, the final data extraction sheet and analysis plan are unable to be finalised until after the review is completed. Documentation of all studies that are not to be included in the data extraction, as per consensus by reviewers, will be listed in a separate sheet for tracking purposes. Abstracted qualitative data will be synthesised using narrative description based on themes identified once the data have been extracted.

Assessment of study quality is not required for scoping reviews ${ }^{19}$ and studies will be included regardless of study quality.

\section{DISSEMINATION AND ETHICS}

To our knowledge, no other published work has systematically synthesised the global health workforce in maternal and newborn care in the context of SAB measurement. We aim to provide a broad and comprehensive review of all available literature with the aim of verifying country-level definitions and functions of SAB in LMIC, specifically related to what their education and training is, what their skills and competencies are and if they are legislated and regulated to perform these functions. It will also identify gaps in the research literature where further academic study is warranted. Once completed, this review will be the foundation for the compilation of a metadata of cadres considered SAB, used to harmonise global measurement of coverage and reporting of this indicator moving forward.

The results from this study will be disseminated through a peer-reviewed publication and will contain supporting documentation on data compiled and analysed for the scoping review. Since a scoping review collects and examines data from existing available literature, this study does not require ethics approval.

Contributors DC is the guarantor of this review. DC, LS and AJH were responsible for the initial conception and design of this study. DC, LS, AJH, ABM, LCA and AA contributed to the development of the eligibility and data extraction criteria. AJH developed and executed the search strategy. AJH, ABM, LCA and DC are responsible for abstract and full-text screening. AJH drafted and revised the manuscript. DC, LS, AJH, ABM, LCA and AA contributed and approved the final version of this protocol.

Funding This work was supported by a subgrant from the Johns Hopkins Bloomberg School of Public Health (JHSPH) with funds provided by Grant No OPP 1096225 from the Bill and Melinda Gates Foundation and the Department of Reproductive Health and Research (RHR), including the UNDP/UNFPA/UNICEF/WHO/ World Bank Special programme of research, development and research training in human reproduction (HRP).

Competing interests None declared.

Provenance and peer review Not commissioned; externally peer reviewed.

Open Access This is an open access article distributed under the terms of the Creative Commons Attribution-NonCommercial IGO License (CC BY-NC 3.0 IG0), which permits use, distribution, and reproduction for non-commercial purposes in any medium, provided the original work is properly cited. In any reproduction of this article there should not be any suggestion that WHO or this article endorse any specific organization or products. The use of the WHO logo is not permitted. This notice should be preserved along with the articles original URL. See:

\section{https://creativecommons.org/licenses/by-nc/3.0/igo}

(c) Article author(s) (or their employer(s) unless otherwise stated in the text of the article) 2017. All rights reserved. No commercial use is permitted unless otherwise expressly granted.

\section{REFERENCES}

1. Gaffey MF, Das JK, Bhutta ZA. Millennium Development Goals 4 and 5: Past and future progress. Semin Fetal Neonatal Med 2015;20:285-92.

2. Alkema L, Chou D, Hogan D, et al. Global, regional, and national levels and trends in maternal mortality between 1990 and 2015, with scenario-based projections to 2030: a systematic analysis by the UN Maternal Mortality Estimation Inter-Agency Group. Lancet 2016;387:462-74.

3. Alkema L, New JR, Pedersen J, et al. Child mortality estimation 2013: an overview of updates in estimation methods by the United Nations Inter-agency Group for Child Mortality Estimation. PLoS One 2014;9:e101112.

4. World Health Organization. The world health report 2006 - working together for health: World Health Organization, 2006.

5. Every Woman Every Child, World Health Organization. The Global Strategy for Women's, Children's, and Adolescent's Health (20162030): Survive, Thrive, Transform. New York, NY, USA: United Nations, 2015. 
6. United Nations. Sustainable Development Goals. New York: United Nations, 2016. http://www.un.org/sustainabledevelopment/ sustainable-development-goals/. (Accessed 1 November 2016).

7. United Nations Statistics Division. Metadata for the Proposed Global Indicators for the Review of the 2030 Agenda for Sustainable Development: Metadata for Goal 3. http://unstats.un.org/sdgs/files/ metadata-compilation/Metadata-Goal-3.pdf.

8. Every Woman Every Child, World Health Organization. Indicator and monitoring framework for the global strategy for women's, children's, and adolescents' health (2016-2030): Every Women Every Child, 2016.

9. Moran AC, Jolivet RR, Chou D, et al. A common monitoring framework for ending preventable maternal mortality, 20152030: phase I of a multi-step process. BMC Pregnancy Childbirth 2016;16:250.

10. Moxon SG, Ruysen H, Kerber KJ, et al. Count every newborn; a measurement improvement roadmap for coverage data. $B M C$ Pregnancy Childbirth 2015;15 Suppl 2:S8.

11. World Health Organization. Making pregnancy safer: the critical role of the skilled attendant A joint statement by WHO, ICM and FIGO. World Health Organization 2004.

12. Adegoke A, Utz B, Msuya SE, et al. Skilled Birth Attendants: who is who? A descriptive study of definitions and roles from nine Sub Saharan African countries. PLoS One 2012;7:e40220.

13. Ueno E, Adegoke AA, Masenga G, et al. Skilled birth attendants in Tanzania: a descriptive study of cadres and emergency obstetric care signal functions performed. Matern Child Health $J$ 2015;19:155-69.

14. Deller B, Tripathi V, Stender S, et al. Task shifting in maternal and newborn health care: key components from policy to implementation. Int J Gynaecol Obstet 2015;130:S25-S31.

15. Harvey SA, Blandón YC, McCaw-Binns A, et al. Are skilled birth attendants really skilled? A measurement method, some disturbing results and a potential way forward. Bull World Health Organ 2007;85:783-90.

16. Arksey H, O'Malley L. Scoping studies: towards a methodological framework. Int J Soc Res Methodol 2005;8:19-32.
17. Bragge P, Clavisi O, Turner T, et al. The Global Evidence Mapping Initiative: scoping research in broad topic areas. BMC Med Res Methodol 2011;11:92.

18. Levac D, Colquhoun H, O'Brien KK. Scoping studies: advancing the methodology. Implement Sci 2010;5:69.

19. Peters MD, Godfrey CM, Khalil H, et al. Guidance for conducting systematic scoping reviews. Int J Evid Based Healthc 2015;13:141-6.

20. Moher D, Liberati A, Tetzlaff J, et al. Preferred reporting items for systematic reviews and meta-analyses: the PRISMA Statement. Open Med 2009;3:e123-30.

21. Liberati A, Altman DG, Tetzlaff J, et al. The PRISMA statement for reporting systematic reviews and meta-analyses of studies that evaluate health care interventions: explanation and elaboration. PLoS Med 2009;6:e1000100.

22. ICF International. The Demographic and Health Surveys (DHS) Program, 2016. http://www.dhsprogram.com/. (Accessed 1 November 2016).

23. UNICEF. Statistics and Monitoring: Multiple Indicator Cluster Surveys (MICS). 2016. http://mics.unicef.org. (Accessed 1 November 2016).

24. United Nations Statistics Division. Sustainable Development Goals (SDG) Indicators, Global Database. 3.1.2: Proportion of births attended by skilled health personnel. https://unstats.un.org/sdgs/ indicators/database/?indicator=3.1.2

25. Footman K, Benova L, Goodman C, et al. Using multi-country household surveys to understand who provides reproductive and maternal health services in low- and middle-income countries: a critical appraisal of the Demographic and Health Surveys. Trop Med Int Health 2015;20:589-606.

26. Landis JR, Koch GG. The measurement of observer agreement for categorical data. Biometrics 1977;33:159-74.

27. World Health Organization, UNFPA, UNICEF and Mailman School of Public Health Averting Maternal Death and Disability. Monitoring emergency obstetric care: A handbook: World Health Organization, 2009.

28. World Health Organization, United Nations Population Fund, UNICEF, The World Bank. Managing complications in pregnancy and childbirth: A guide for midwives and doctors. World Health Organization 2003. 